\title{
OPEN Cellular pathways during spawning induction in the starlet sea anemone Nematostella vectensis
}

\author{
Shelly Reuven ${ }^{1,3}$, Mieka Rinsky ${ }^{2,3}$, Vera Brekhman ${ }^{1}$, Assaf Malik ${ }^{1}$, Oren Levy ${ }^{2 \bowtie} \&$ \\ Tamar Lotan ${ }^{1 凶}$ \\ In cnidarians, long-term ecological success relies on sexual reproduction. The sea anemone \\ Nematostella vectensis, which has emerged as an important model organism for developmental \\ studies, can be induced for spawning by temperature elevation and light exposure. To uncover \\ molecular mechanisms and pathways underlying spawning, we characterized the transcriptome of \\ Nematostella females before and during spawning induction. We identified an array of processes \\ involving numerous receptors, circadian clock components, cytoskeleton, and extracellular transcripts \\ that are upregulated upon spawning induction. Concurrently, processes related to the cell cycle, fatty \\ acid metabolism, and other housekeeping functions are downregulated. Real-time qPCR revealed \\ that light exposure has a minor effect on expression levels of most examined transcripts, implying \\ that temperature change is a stronger inducer for spawning in Nematostella. Our findings reveal \\ the potential mechanisms that may enable the mesenteries to serve as a gonad-like tissue for the \\ developing oocytes and expand our understanding of sexual reproduction in cnidarians.
}

Sexual reproduction is the predominant mode of procreation in almost all eukaryotes, from fungi and plants to fish and mammals. It generates the conditions for sexual selection, which is a powerful evolutionary force driving morphological, physiological, and behavioral changes in many species ${ }^{1,2}$. Sex is thought to have arisen once and to have been present in the last eukaryotic common ancestor ${ }^{3-5}$; therefore, it is an important trait in evolutionary biology. An informative group of animals for studying evolution are cnidarians, as they are basal metazoans and considered to be a sister group of the Bilateria ${ }^{6}$. Within cnidarians, the anthozoans, a group that includes corals and sea anemones, are among the morphologically simplest extant eumetazoans. Although they can reproduce asexually, their long-term ecological success relies on sexual reproduction ${ }^{7,8}$.

The most well-known sexual reproduction phenomenon in cnidarians is the mass spawning of corals. In this process, which has been observed worldwide, individuals from the same species synchronously release their eggs and sperm to the water column, where fertilization occurs ${ }^{9-11}$. The synchronized spawning is postulated to be controlled by the circadian clock mechanism, which is based on transcription-translation feedback loops and maintains a free-running period of approximately $24 \mathrm{~h}^{12}$. This allows organisms to anticipate and adjust to daily and seasonal environmental cycles ${ }^{13,14}$. The circadian clock obtains environmental cues such as sea temperature, lunar phase, and the daily light cycle ${ }^{11,15,16}$ that activate internal cascades, eventually leading to spawning ${ }^{9,17-19}$. Orthologs of circadian genes, such as Bmal, Clock, Cry1, Cry2, and Timeless, have been identified in most cnidarian genomes ${ }^{20-22}$.

The starlet sea anemone, Nematostella vectensis, has become an important model organism for developmental and evolutionary studies, particularly for comparative studies of the conservation of developmental processes and signaling pathways in cnidarians versus bilaterians ${ }^{23,24}$. Its genome has been sequenced ${ }^{25}$, and spawning can be induced in the lab throughout the year by temperature elevation and light exposure ${ }^{26-28}$. Nematostella polyps can be either male or female and can reproduce asexually by transverse fission or sexually by releasing gametes into the water column, where fertilization occurs ${ }^{29,30}$. As most anthozoans, Nematostella lack real ovaries and the mesenteries serve as gonads, where oocytes develop and mature ${ }^{23}$, leading to changes in tissue shape ${ }^{31}$. The oocytes first appear at the base of the mesenterial gastrodermis and develop asynchronously into the mesoglea while keeping contact with the gastrodermis through the trophonema ${ }^{32,33}$. However, the natural sexual reproduction of Nematostella is still unexplored and it is currently unknown whether it is controlled by the circadian clock or by other mechanisms ${ }^{28}$.

${ }^{1}$ Marine Biology Department, The Leon H. Charney School of Marine Sciences, University of Haifa, 3498838 Haifa, Israel. ${ }^{2}$ Mina and Everard Goodman Faculty of Life Sciences, Bar-llan University, 52900 Ramat Gan, Israel. ${ }^{3}$ These authors contributed equally: Shelly Reuven and Mieka Rinsky. ${ }^{\bowtie}$ email: oren.levy@biu.ac.il; lotant@univ.haifa.ac.il 
Here, we applied comparative transcriptomic methods to reveal the potential cellular and molecular pathways underlying spawning in Nematostella. By characterizing the transcriptome of Nematostella females before and during the different stages of spawning induction, we identified an array of upregulated processes involving numerous receptors as well as extracellular matrix (ECM) organization and regulation of actin, along with downregulated processes related to cell cycle, fatty acid metabolism and other housekeeping functions. The regulation of these processes sheds light on the possible mechanisms that may enable the mesenteries to serve as a gonad-like tissue for the developing oocyte.

\section{Results}

Transcriptomic analysis during spawning induction. To understand the molecular pathways leading to the release of oocytes in Nematostella, females were induced by temperature elevation and light exposure (see "Methods") and then sampled for RNA-Seq analysis at 1, 2, 5, and $8 \mathrm{~h}$ during induction in addition to a pre-induction control sampling (Fig. 1A). Transcriptome sequencing produced an average of 16.5 million raw reads per sample, filtered and mapped to the N. vectensis genome (NCBI genome assembly GCA_000209225.1 ASM20922v1). Based on FPKM (fragments per kilobase million) normalized read counts per transcript per sample, we estimated the distance between samples using principal component analysis (PCA) ordination, which demonstrates that although individual variance at each time point exists among the samples, the distance between the baseline and the treated samples grows with time during induction (Fig. 1B). Generalized linear model in DESeq2 revealed $\sim 1500$ transcripts that were found to be differentially expressed $($ FDR $<0.05)$ during spawning induction, as compared to pre-induction levels (Fig. 1C, Supplementary Table S1). MA plots (Fig. 1D-G) demonstrate that during the induction process, both the number of differentially expressed transcripts and their fold-change magnitudes are gradually increased. This gradual effect is also seen in a heatmap, which additionally shows two distinct groups with opposite trends (Fig. 1C). The first group includes 608 transcripts that were upregulated $8 \mathrm{~h}$ after induction began, whereas the second group includes 628 transcripts that were downregulated $8 \mathrm{~h}$ into induction.

Gene ontology (GO) enrichment analysis. Next, we analyzed the enriched pathways of the differentially expressed transcripts during the experiment timeline. GO analysis revealed that $88 \%$ of the significantly enriched upregulated biological processes and molecular function categories were either specific to the first hour of induction $(n=117,42 \%)$ or shared between two or more time points $(n=126,46 \%)$ (Fig. 2, Supplementary Table S2). Processes overrepresented among upregulated transcripts included terms related to light reception and chemical stimuli involving numerous receptors, as well as processes linked to the ECM and the nervous system. Among the significantly enriched downregulated biological processes and molecular function categories, $47 \%(n=168)$ were shared between time points and included terms related to cell cycle, oxidation-reduction, and various metabolic processes (Fig. 2, Supplementary Table S2).

Processes related to light, ECM, and cytoskeleton are upregulated during spawning induction. Since light is one of the triggers of spawning induction in Nematostella ${ }^{26}$, we analyzed the GO term sensory perception of light stimulus (GO:0050953), which was upregulated during the first hour of induction (Figs. 2, 3A). The analysis revealed that transcripts associated with this term are expressed mainly at $1 \mathrm{~h}$ and $8 \mathrm{~h}$ into induction and that many are receptors, specifically G protein-coupled receptors (GPCRs) (Fig. 3A). Moreover, 5 and $8 \mathrm{~h}$ into induction, there was an upregulation of transcripts involved in photoreceptor activity (GO:0009881) (Figs. 2, 3B), and $8 \mathrm{~h}$ into induction, there was also an upregulation of the photoperiodism process (GO:0009648), though not upholding significance $(p=0.05)$. Together, these two processes regulated 11 transcripts, five of which are core components of the circadian clock. Among the circadian clock transcripts, Clock and three cryptochromes (Cryla, Cry $1 b$, and Cry) were downregulated before induction and their expression elevated during the process, peaking after $8 \mathrm{~h}$. The transcript Cry 1 displayed an opposite expression trend, as it was upregulated before induction started and decreases during the process (Fig. 3B). Other transcripts that are related to photoreceptor activity, such as melanopsin, rhodopsin, protease usp6 $\mathrm{n}$-terminal-like, rhodopsinlike GPCR and opsin BCRH2-like, displayed a similar expression trend of upregulation during the induction process.

We also analyzed the term ligand-gated ion channel activity (GO:0015276), which was common to all sampling time points (Figs. 2, 3C). The analysis revealed four different $\mathrm{GABA}_{\mathrm{A}}$ receptor subunits and three transcripts related to the excitatory neurotransmitter glutamate. Two of the glutamate-related transcripts $(171792,132356)$ displayed similar patterns of upregulation, mainly at the first hour of induction, whereas the third transcript (138860) was strongly upregulated at the eighth hour of induction (Fig. 3C).

We then used STRING to analyze the cytoskeleton and ECM proteins related to the GO terms actin filamentbased movement (GO:0030048), extracellular matrix structural constituent (GO:0005201), and positive regulation of actin filament polymerization (GO:0030838), which were upregulated at the first, second, and eighth hour of the induction process, respectively. The analysis revealed a complex network that can be divided into three main groups: ECM, actin cytoskeleton, and microtubule cytoskeleton (Fig. 3D). Some of the microtubule isoforms were upregulated, whereas others were downregulated and displayed a weak connection to isoforms of motor proteins, such as dynein and kinesin (Fig. 3D). The myosin motor proteins are connected to actin, which showed strong connections to three GTPases: RhoA, Cdc42, and Rac1. While the first two were upregulated, Rac1 was downregulated. Additionally, connected to actin is the integrin receptor Itgb1, which was downregulated and strongly connected to different isoforms of collagens comprising the ECM network, all found to be upregulated (Fig. 3D). Overall, the upregulated processes indicate that spawning induction is perceived by different receptors, some of which are light-sensitive receptors that initiate signaling cascades and cytoskeletal rearrangement. 
Induction time (h)

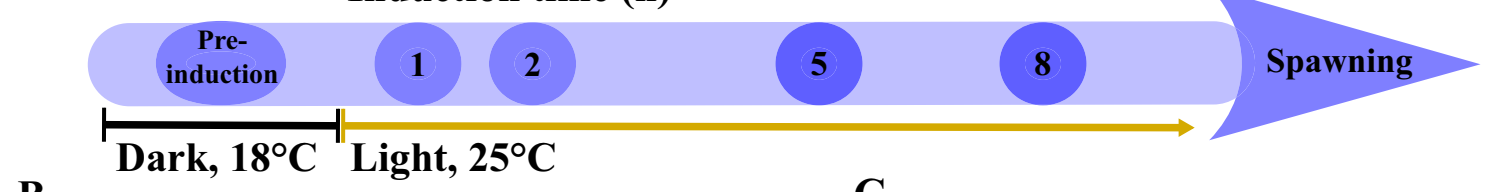

B
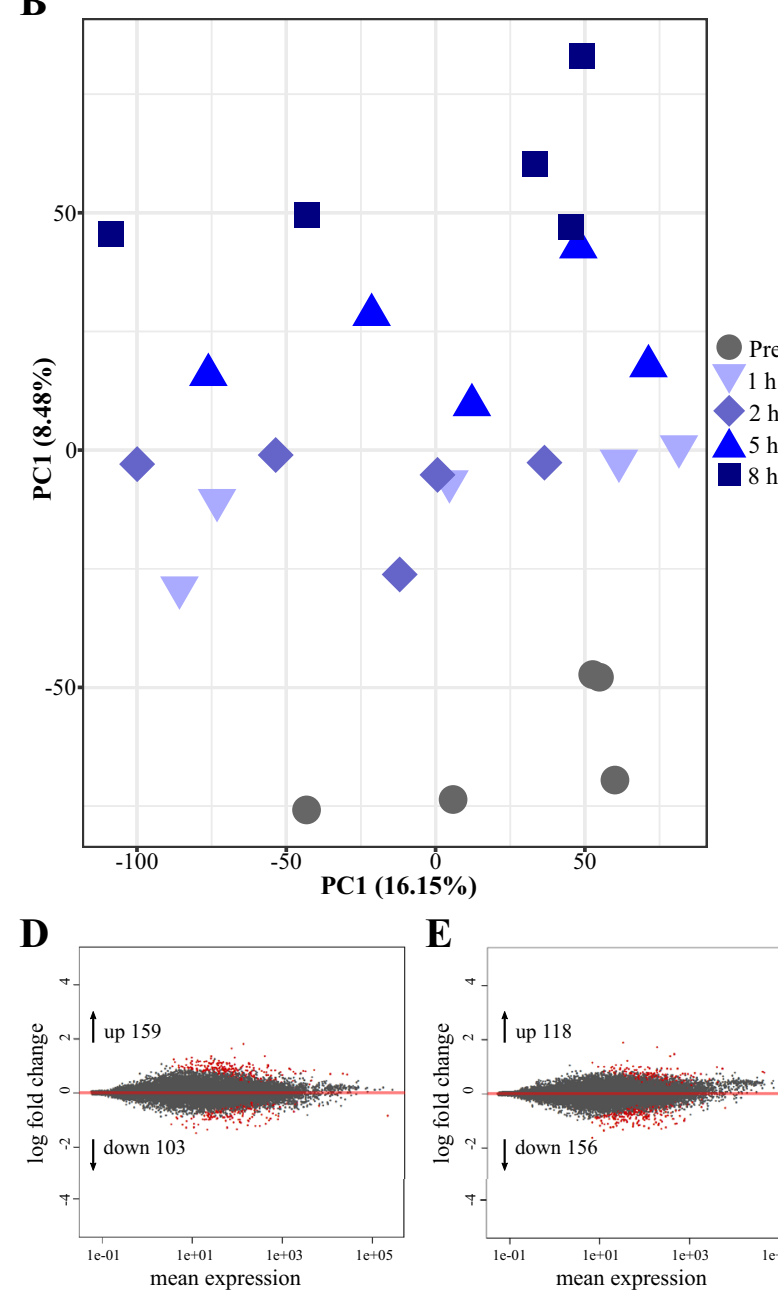

E

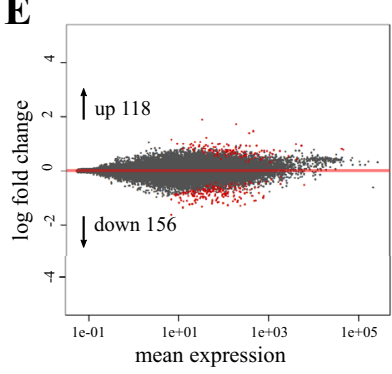

C

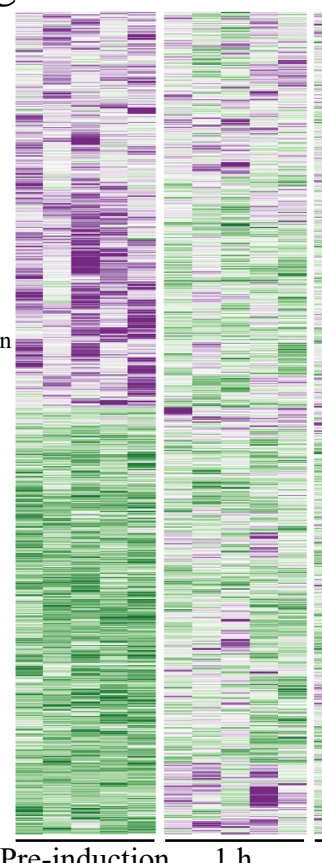

Pre-induction $1 \mathrm{~h}$

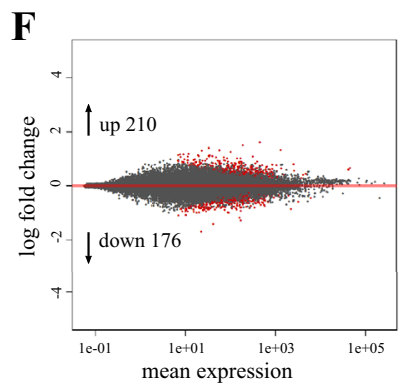

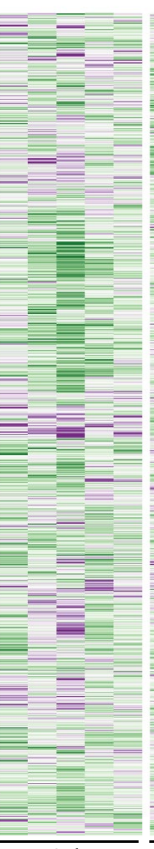

$2 \mathrm{~h}$
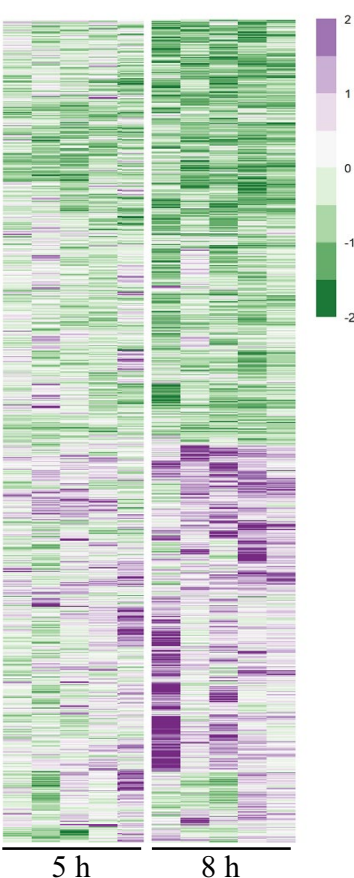

$\mathbf{G}$

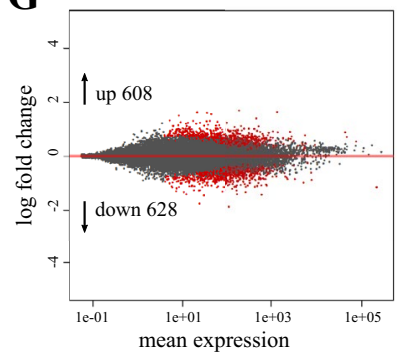

Figure 1. Transcriptome analysis of Nematostella females before and at different time points during spawning induction. (A) Schematic presentation of the transcriptome experiment. Induction time refers to time of temperature and light stimulus to successful spawning. Sampling time points are indicated in circles. Dark $18^{\circ} \mathrm{C}$ (pre-induction) and light $25^{\circ} \mathrm{C}$ (induction) regimes are indicated by black and yellow lines. (B) Principal component analysis (PCA) ordination indicates a time-dependent clustering of samples. Color-coded numbers on the right indicate sampling time points $(n=5$ individuals per time point). (C) Heatmap of differential expressed genes at pre-induction and at the four sampling time points during induction. Rows represent transcripts, columns represent the tested individuals $(n=5)$. Expression level is indicated by $\mathrm{z}$-score. The full list can be found in Supplementary Table S1. (D-G) MA plots showing differentially expressed transcripts at $1 \mathrm{~h}$ (D), $2 \mathrm{~h}(\mathbf{E}), 5 \mathrm{~h} \mathrm{(F),} \mathrm{and} 8 \mathrm{~h}(\mathbf{G})$ after induction began, compared to pre-induction. The numbers of up- and down-regulated transcripts are indicated within each plot. Y-axis represents the $\log _{2}$ fold change, and the $\mathrm{X}$-axis represents mean normalized read counts. Red dots represent differentially expressed transcripts.

Metabolic processes are downregulated during spawning induction. A large group of downregulated processes included seven terms associated with metabolism. Only three of these processes were shared among time points, two of which are related to lipid metabolism, namely fatty acid metabolic process and neutral lipid metabolic process (Fig. 2). KEGG analysis of the term fatty acid metabolic process (GO:0006631) revealed that nine transcripts function in fatty acid degradation, and except one, all of them were downregulated (Supplementary Fig. S1). These results indicate that the degradation of fatty acid and consequently the formation of acetyl-CoA, which is required for ATP production, are reduced during spawning induction. 


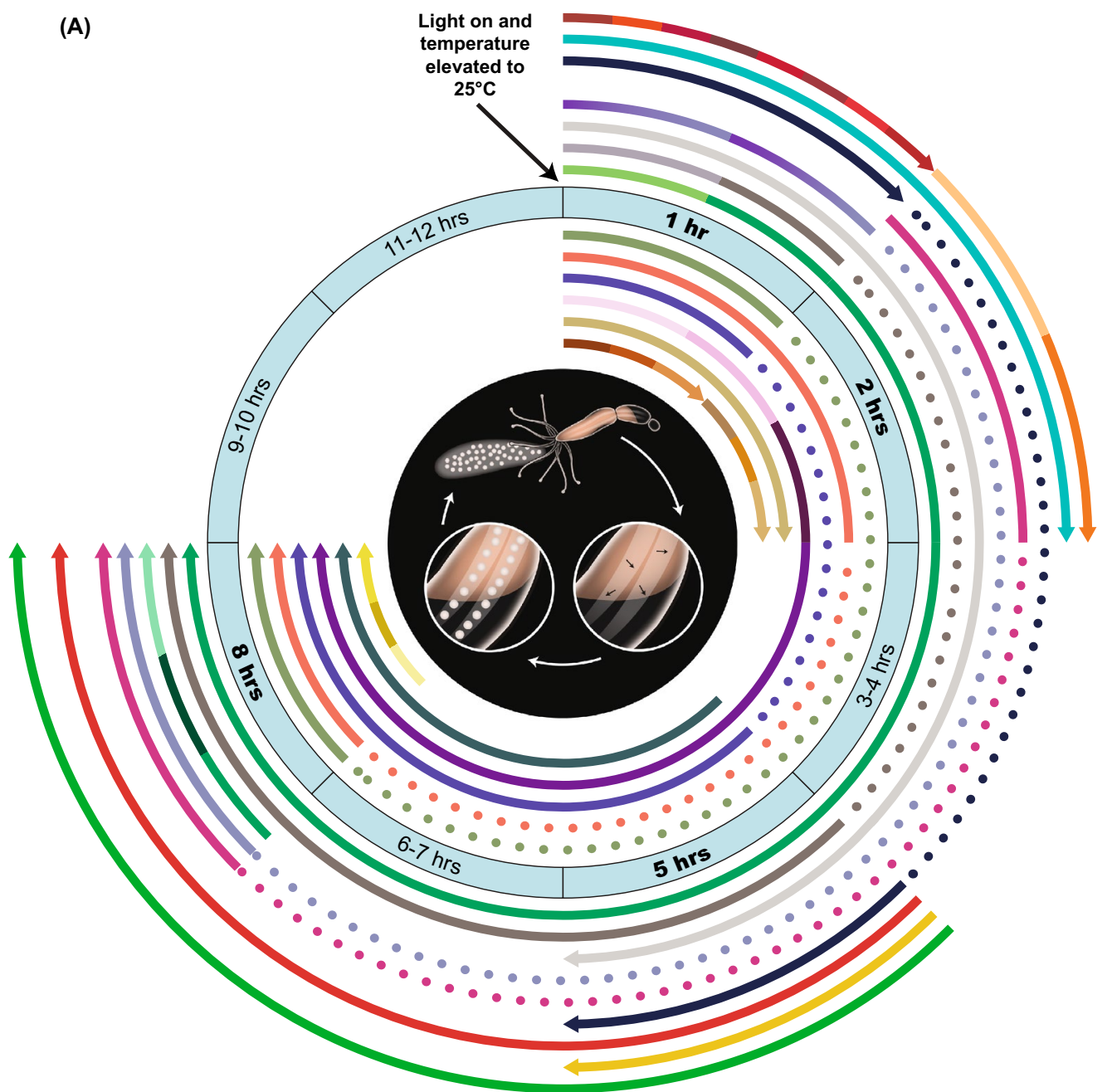

(B) Upregulated GO terms

(C) Downregulated GO terms

\begin{tabular}{|c|c|c|}
\hline \multirow{2}{*}{\begin{tabular}{|c|c|c|c|}
\multicolumn{3}{|c}{ Hours } \\
1
\end{tabular}} & & Ho \\
\hline & GO term & \\
\hline & Neuropeptide signaling pathway & \\
\hline & Cell recognition & \\
\hline & Stem cell proliferation & \\
\hline & Ionotropic glutamate receptor signaling pathway & \\
\hline & Sensory perception of light stimulus & \\
\hline & Actin filament-based movement & \\
\hline & Fertilization & \\
\hline & Extracellular structure organization & \\
\hline & Acid secretion & \\
\hline & Activation of adenylate cyclase activity & \\
\hline & Epithelial to mesenchymal transition & \\
\hline & Glycoprotein biosynthetic process & \\
\hline & Positive regulation of actin filament polymerization & \\
\hline & Amine transport & \\
\hline & Neurotransmitter transport & \\
\hline & Calcium ion homeostasis & \\
\hline & Phototransduction & \\
\hline & Detection of chemical stimulus & \\
\hline & Structural constituent of ribosome & \\
\hline & Photoreceptor activity & \\
\hline & $\begin{array}{l}\text { G-protein coupled peptide receptor activit } \\
\text { Synaptic transmission, glycinergic }\end{array}$ & \\
\hline & Detection of stimulus & \\
\hline & $\mathrm{C}$ & \\
\hline
\end{tabular}

\section{Hours}

\section{GO term}

\section{Cofactor metabolic process}

Protein folding

RNA splicing

- Macromolecule methylation

Microtubule cytoskeleton organization

Fatty acid derivative biosynthetic process

Regulation of catabolic process

Lipid modification

DNA-templated transcription

Regulation of DNA metabolic process

Mitochondrion organization

- Neutral lipid metabolic process

Cell cycle phase transition

Ribosom biogenesis

Fatty acid metabolic process

DNA replication

Oxidation-reduction process 
4Figure 2. Infographic cartoon presenting temporal distribution from representative GO terms during spawning induction. (A) The middle circle indicates the timeline of induction, from light and temperature change to gamete release. Sampling time points are marked in bold. Each arc indicates the start point and endpoint (arrowhead) of up- or downregulated GO terms, which are shown in different colors; dashed arcs indicate temporary pauses in enrichment for the GO terms. Outer arcs represent upregulated enriched GO terms, and inner arcs represent enriched downregulated GO terms. Illustration in the inner black circle shows the main stages of the induction process: oocytes develop in mesenteries, leading to the release of the egg sack into the water after approximately $12-15 \mathrm{~h}$. (B) Upregulated and (C) downregulated representative GO terms at $1 \mathrm{~h}, 2 \mathrm{~h}$, $5 \mathrm{~h}$, and $8 \mathrm{~h}$ during spawning induction. The same color scheme as in $\mathrm{A}$ is used, and the color of each term is annotated. The complete list of all GO terms can be found in Supplementary Table S2.

Light effect on the induction process. Next, we performed real-time qPCR on selected transcripts obtained from RNA-seq to test their transcriptional response to light during induction. For that, Nematostella females were induced by temperature elevation under either light or dark conditions and were sampled at the same time points as in the RNA-seq experiment (Supplementary Fig. S2A). While in both groups spawning rates were about $80 \%$, some transcripts displayed different expression patterns in response to light (Supplementary Fig. S2). Out of seven clock-related transcripts examined, five transcripts (Cry1a, Crylb, Clock, BHLH transcription factor Helt, and glutamate receptor Grin1) were upregulated during light induction and at the eighth hour of the dark induction, whereas one transcript, thyrotroph embryonic factor (Tef), was downregulated as induction proceeded under both light and dark conditions (Supplementary Fig. S2B-H). We additionally tested two cytoskeleton-related transcripts, tubulin 1 (Tuba1) and Wnt3, and the aquaporin (Aqp) water-selective channel. With the exception of $A q p$, no significant differences in expression levels were found between the two treatment groups (Supplementary Fig. S2I-K). These results support the findings of light perception during spawning induction but also demonstrate that temperature raise is a stronger signal for spawning.

\section{Discussion}

To explore the molecular mechanisms and pathways underlying spawning induction in the basal metazoan Nematostella, we analyzed the transcriptome of females before and during the induction process. Our results revealed a diverse array of up-and-downregulated processes, which lead to successful spawning. Furthermore, a complementary light/dark induction experiment revealed that the expression levels of most tested transcripts changed significantly under both conditions, demonstrating that temperature change is a stronger signal for spawning. These molecular data support the predominance of temperature change over light, which was shown before in spawning experiments ${ }^{26}$.

In cnidarians, light has been shown to have a fundamental role in regulating sexual reproduction. The spawning of the hydromedusa Clytia hemisphaerica and Cladonema pacificum is triggered by light-dark transition and is mediated by opsin photoreceptors from the GPCR family ${ }^{34-36}$. In the corals Acropora millepora and Acropora digitifera, the photopigment melanopsin, which belongs to the opsin family, is upregulated during the spawning night, possibly triggering different signaling cascades contributing to gamete release ${ }^{17,19}$. We found upregulation of several GO terms related to light reception and transduction as well as to GPCR and ion channel activity, including melanopsin and rhodopsin receptors from the GPCR family and a glutamate receptor. This suggests that in Nematostella, light-sensitive proteins are responsible for receiving the light signal and initiate different cascades, such as GPCR signaling and glutamate release. Additionally, our qPCR analysis showed that the glutamate receptor Grin1, an ion-gated channel ${ }^{37,38}$, was upregulated at the eighth hour of both light and dark inductions. Previous studies have shown that the glutamate pathway interacts closely with the molecular feedback loops of the circadian clock ${ }^{39,40}$. In A. digitifera and A. millepora, upregulation of transcripts related to glutamate release by photoreceptors was observed during the spawning event ${ }^{17,19}$. Together, these results suggest a conserved role for GPCR and glutamate signal transduction during the reproduction process of Nematostella. However, the exact mechanism is yet to be uncovered.

The core components of the circadian clock, cryptochromes and Clock, have been identified in Nematostella and were shown to have diel rhythmicity. Moreover, there is evidence that Nematostella exhibits circadian behavior and physiology under diel light cycles that are maintained upon the removal of light ${ }^{20,41-43}$. In A. millepora, two cryptochromes that displayed rhythmic gene expression under light:dark cycles were suggested to play a role in synchronizing spawning ${ }^{18}$. Our results showed that the expression of Cryla, Crylb, and Clock was upregulated during light induction, while in the dark induction, they were upregulated at the eighth hour only. Since cultivated cultures of Nematostella can be induced in light and dark conditions, our results suggest that Nematostella Crys' and Clock are not essential for initiating the process yet, may play non-circadian roles later during spawning induction. Another clock-related gene, Tef, which belongs to the PAR family of transcription factors, was shown to be expressed in vertebrates with a circadian rhythms ${ }^{44}$ and to regulate downstream light-induced genes under circadian control in zebrafish ${ }^{45}$. Our results demonstrated that in Nematostella, Tef is not affected by light, as it was downregulated in both light and dark conditions. This is in line with the findings in A. millepora, where Tef was downregulated prior to and during spawning ${ }^{17}$. It is possible that Tef functions as a repressor of a yet unknown pathway and therefore its downregulation allows the pathway to operate. Although the circadian clock plays an important role in the reproduction of many organisms from different phyla ${ }^{46-49}$, our results indicate that spawning of Nematostella, during controlled laboratory inductions, is most likely not regulated by the endogenous clock. Notably, the circadian expression pattern of these clock genes is strongly dependent on consistent light:dark cycles ${ }^{42,50}$; however, the studied sea anemones were maintained in constant temperature 
A

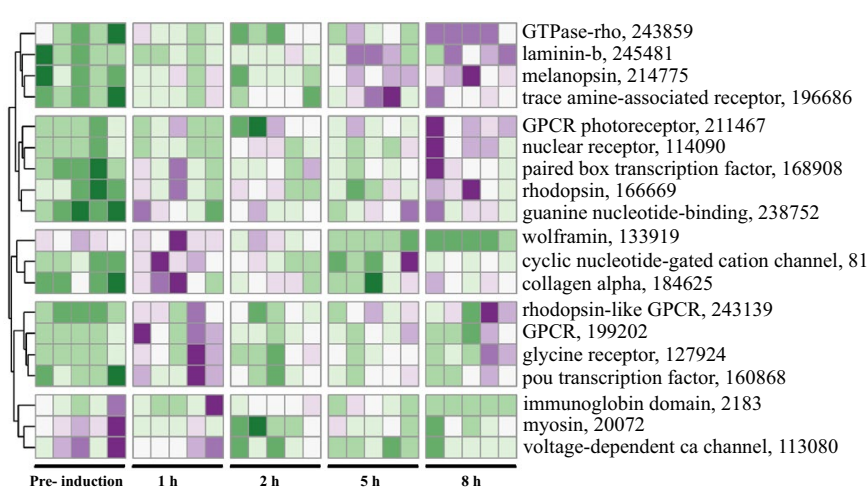

C

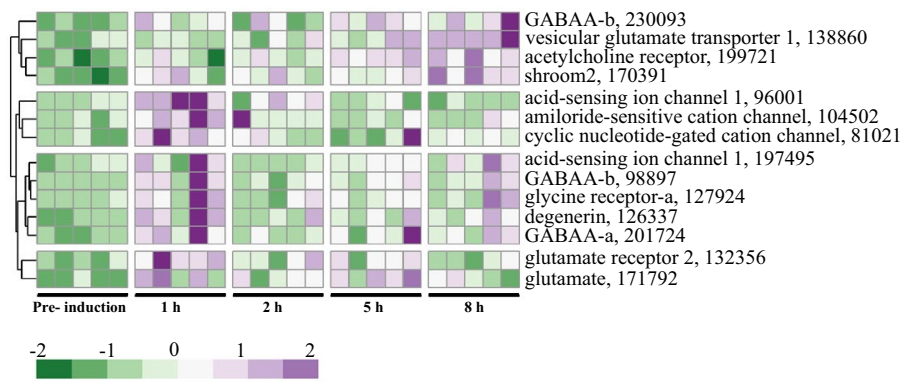

B

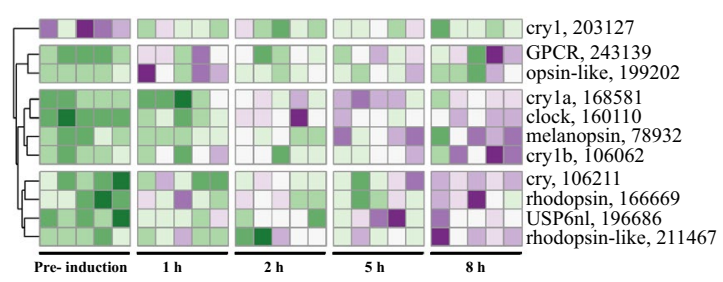

D

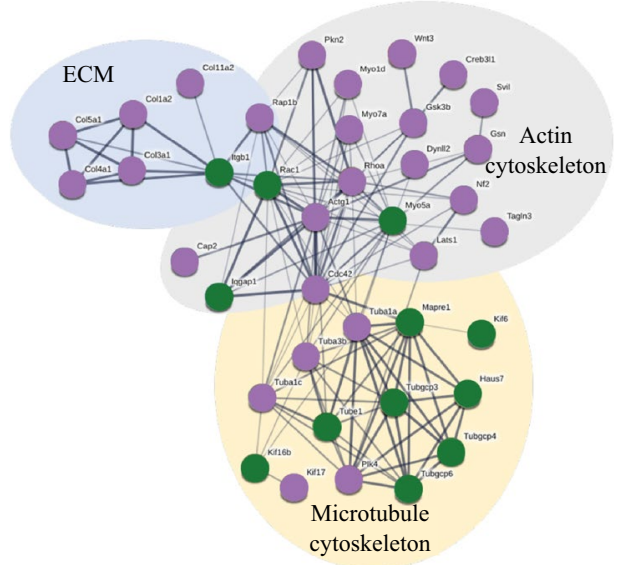

Figure 3. Analysis of transcripts related to light, ion channel, ECM, and cytoskeleton. Heatmap of transcript expression related to (A) sensory perception of light stimulus (GO:0050953), (B) photoreceptor activity (GO:0009881) and photoperiodism (GO:0009648) and (C) ligand-gated ion channel activity (GO:0015276) at the different sampling time points during induction, relative to the pre-induction control (FDR $<0.05)$. Rows represent transcripts, columns represent the tested individuals ( $\mathrm{n}=5$ per time point). Expression level is indicated by z-score. Numbers are JGI IDs. (D) STRING visualization of protein interactions of cytoskeletonand ECM-related transcripts. Upregulated transcripts are in purple and downregulated in green. The proteins presented in the network are mouse homologs. Thickness of the connecting lines indicates the strength of data support.

and darkness for a long period and were not entrained by daily exogenous cues. Therefore, more investigation is required to understand the role of circadian clock components during spawning induction in Nematostella.

One of the expected changes during spawning induction is the rearrangement of cytoskeletal networks. In the corals Acropora gemmifera, A. millepora, and A. digifera, cytoskeletal reorganization was elevated during and after spawning ${ }^{17,19,51}$. Our results demonstrated that, likewise, extensive changes in the cytoskeleton and its related proteins occur during spawning induction in Nematostella. We have discovered transcripts that are involved in actin regulation, such as the small GTPases RhoA, Rac1, and $C d c 42$ (Fig. 4). Cdc42 promotes the formation of filopodia ${ }^{52}$ and RhoA regulates the formation of linear actin network, focal adhesion and stress fibers, which are the major contractile structures required for cell adhesion during migration ${ }^{53,54}$. When $R h o A$ is activated, it affects the actomyosin contractile network ${ }^{55}$. This stands in line with our results showing upregulation of RhoA and myosins. Rac1 controls the formation of branched actin network and the formation of lamellipodia in the leading edge of motile cells $s^{54}$. RhoA and Racl usually antagonize each other ${ }^{56}$ and the balance between them was shown to control cell shap ${ }^{57}$. The finding of upregulation of RhoA and downregulation of Racl suggests that such balance may allow the mesenteries to change shape and create space for oocyte development. Additionally, it was shown that in migrating cells, branched actin network is localized in the leading edge of the cell, while stress fibers are present in the cell body, anchoring it to the surface through focal adhesion $s^{58}$. Our findings indicate a similar actin organization, suggesting that cell migration occurs during spawning induction.

The ECM serves as a substrate for cell migration and transfers signals into the cell from the surrounding environment through adhesion receptors such as integrins, which promote reorganization of the cytoskeleton ${ }^{59,60}$. In addition, ECM remodeling plays a crucial part in regulating diverse cellular processes such as cell shape and growth $^{61}$. Our results showed upregulation of many different isoforms of collagen, the most abundant ECM proteins $^{62}$ (Fig. 4), possibly indicating ECM rearrangement.

Many of the downregulated GO terms we found are related to metabolic processes, such as fatty acid metabolism. This could imply that during induction, less energy is generated in the mesenteries. In contrast, fatty acid oxidation is increased in the oocyte ${ }^{63}$. We additionally found that transcripts related to cell cycle, an energy consuming process, were downregulated (Fig. 4). Furthermore, DNA, RNA, and protein-related processes were downregulated, suggesting that cell maintenance was reduced. Altogether, these results indicate that there is a decrease in metabolic processes that are not required for spawning. The mesenteries were previously shown to host the synthesis of the fatty acid-rich vitellogenin (Vtg), which is the main yolk protein in Nematostella oocytes $^{31,63}$. In fish, vitellogenesis is accompanied by an increase in fatty acid in the liver, where the process occurs. Decrease in fatty acid in the liver and their increase in gonads were observed before spawning and 


\section{Light and Temperature}

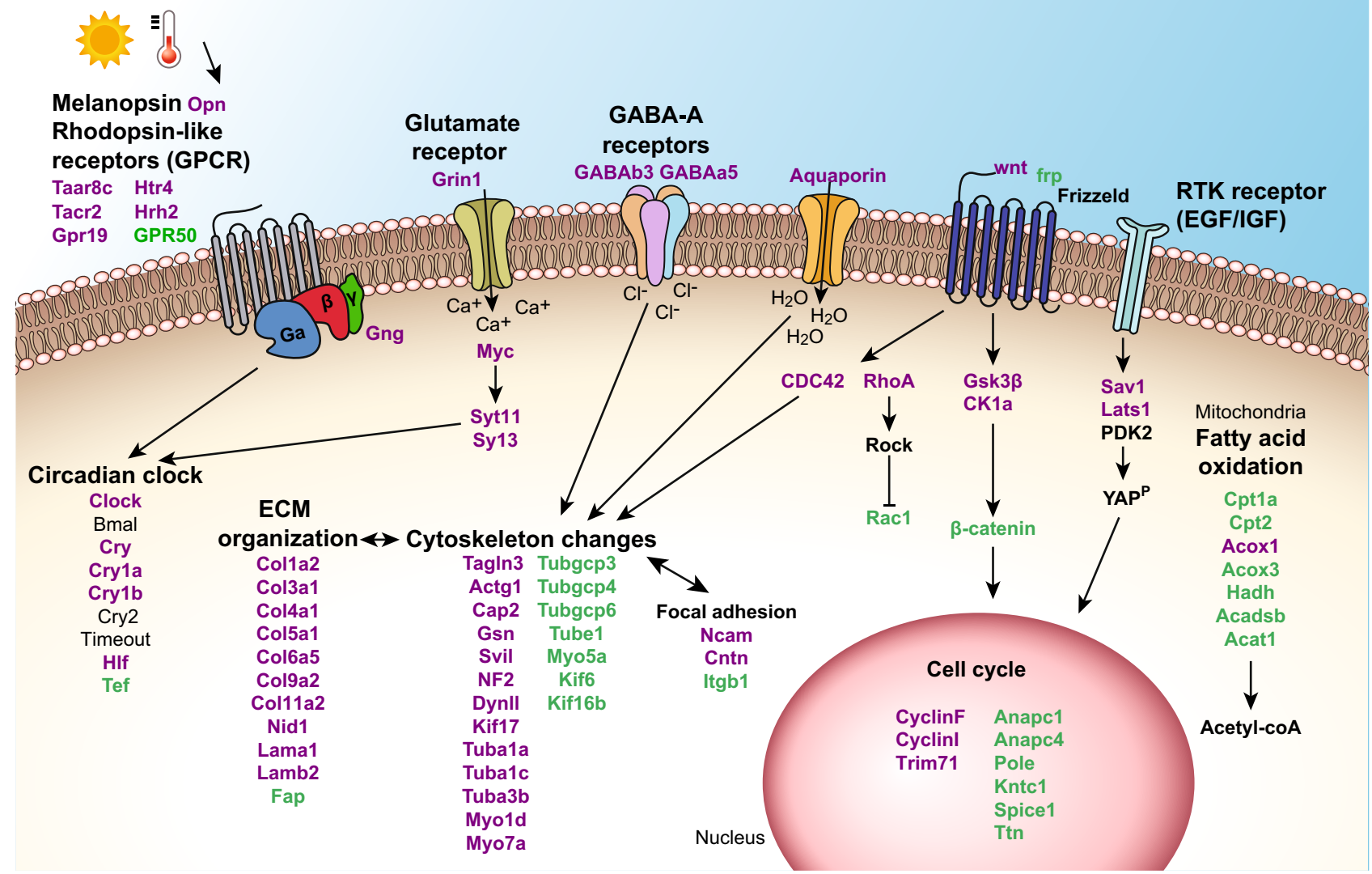

Figure 4. Proposed model for molecular signaling during spawning induction in Nematostella vectensis. The process is triggered by light and temperature elevation. We propose that light stimulates melanopsinlike homologue, which activates rhodopsin and glutamate receptors and initiates GPCR signaling cascades. Other receptors are also activated and together they affect cell cycle, cytoskeleton and ECM organization, focal adhesion, fatty acid oxidation, and circadian clock components. Eventually, these processes lead to successful spawning. Upregulated transcripts are written in purple and downregulated transcripts are in green.

occurred due to transport of Vtg to the oocyte ${ }^{64,65}$. We, therefore, postulate that the observed decrease in fatty acid breakdown might be a result of Vtg synthesis. Together, these findings may possibly indicate that the mesenteries function as gonads during spawning in Nematostella.

To conclude, this study reveals molecular pathways underlying spawning induction in female sea anemones, $N$. vectensis. Our results demonstrate that upon the reception of light and temperature signals, processes involving numerous receptors, circadian clock components, the cytoskeleton, and ECM are upregulated, whereas housekeeping processes are mostly downregulated. As shown by the conceptual model in Fig. 4, we suggest that these changes may enable the role of the mesenteries as a gonad-like tissue for the developing oocyte and eventually lead to spawning. Our findings demystify the progression of spawning induction in basal metazoan and could assist in deeper understanding the evolution of spawning patterns of higher organisms that share a common ancestor with Cnidaria.

\section{Methods}

Nematostella cultivation. Nematostella were kept in artificial seawater (12.5 ppt, pH 8.0, Red Sea, Israel) at $18{ }^{\circ} \mathrm{C}$ in the dark. They were fed five times a week with freshly hatched brine shrimps (Artemia salina) with weekly seawater changes. Spawning was controlled and induced as described ${ }^{26}$. In short, on the day before spawning induction, the anemones were not fed, and spawning was conducted in an incubator under white light by raising the temperature from 18 to $25^{\circ} \mathrm{C}$ for $12 \mathrm{~h}$.

RNA-seq experimental design. For the RNA-seq experiment, 25 well-fed female anemones were collected. As a control, five individual anemones were placed in a dark incubator at $18^{\circ} \mathrm{C}$, sampled before spawning induction, and immediately snap-frozen in liquid nitrogen. Spawning was then induced as described above. At $1,2,5$, and $8 \mathrm{~h}$ during induction, five females per time point were sampled and immediately snap-frozen in liquid nitrogen. Samples were stored at $-80^{\circ} \mathrm{C}$ until processing.

qPCR light/dark experiment. For the light/dark induction RT-qPCR experiment, 60 female anemones were divided into two groups. Whereas 30 females were induced for spawning under the same conditions as in 
the RNA-seq experiment, 30 females were induced in the same incubator that was covered to create dark conditions. In addition to a pre-induction sampling that served as a control for both groups, individual sea anemones from both treatment groups were sampled at the same time points as in the RNA-seq experiment at 1, 2, 5, and $8 \mathrm{~h}$ during induction and frozen immediately. To ensure a proper spawning process, 87 females were induced by temperature and light and 48 by temperature in the dark; $81 \%$ and $77 \%$ of them, respectively, released egg sacks.

RNA extraction. Total RNA was extracted from frozen anemone females using Quick-RNA MiniPrep Kit (Zymo Research, Irvine, USA), according to the manufacturer's instructions with minor modifications. Lysis was performed using $3 \mathrm{~mm}$ glass beads in TissueLyser II (QIAGEN) and additional centrifugation at $1000 \mathrm{~g}$ for 1 min was then performed to precipitate unbroken particles. Genomic DNA residues were removed by Turbo DNase treatment (ThermoFisher Scientific), and final RNA was eluted in $80 \mu$ l nuclease-free ultra-pure water. Purified RNA concentrations were determined using NanoDrop 2000c spectrophotometer (Thermo Scientific). Extracted RNA from the RNA-seq experiment was quality assessed using TapeStation 2200 (Agilent Technologies) and extracted RNA from the qRT-PCR experiment was quality assessed using gel electrophoresis $(1 \%$ agarose). RNA samples were stored at $-80^{\circ} \mathrm{C}$ until use.

Next-generation sequencing and data processing. Total RNA isolated from five females at each sampling point (RNA integrity number RIN $>8$ ) was used for transcriptomic analysis. The 25 RNA samples were prepared using the Illumina TruSeq RNA Library Preparation Kit v3, according to the manufacturer's protocol. The libraries were multiplexed on two lanes of an Illumina HiSeq2000 machine at the Life Sciences and Engineering Infrastructure Center at the Technion, Haifa. Illumina reads data (50 bp, single-end) were qualityfiltered and adapter-trimmed using Trimmomatic 0.30 (90-96\% mapping $)^{66}$. Filtered reads were mapped and quantitated with STAR (version 2.4.2a) ${ }^{67}$ using the N. vectensis genome (NCBI genome GCA_000209225.1). 7-13 million reads per sample were mapped uniquely to the genome assembly. Illumina results were deposited in the SRA database under accession no. PRJNA612671.

Differential expression analysis. Differential expression analysis was conducted using generalized linear models (GLMs) in Bioconductor DESeq2 (in R 3.5.1), considering a single factor: induction time ${ }^{68}$. Differential expression was considered to be significant at p-value $<0.05$ (adjusted using Benjamini-Hochberg correction), and transcript abundance is presented as FPKM values produced in DESeq2 (Supplementary Table S1). MA plots were generated with DESeq2 package in R language using DESeq2 lfcShrink function. Heatmaps of normalized FPKM values of all the significantly expressed transcripts were generated using the heatmap.2 function from package GPLOTS (v2.17.0) in R. Expected factor-dependent trends were verified using Principal component analysis (PCA) graphs in stats (v3.6.2).

Gene ontology (GO) enrichment analysis. Functional enrichment analysis was conducted using Bioconductor GoSeq (in R 3.5.1) ${ }^{69}$, with the Wallenius bias method, to allow correcting for gene-length biases in differential transcript expression. In addition, functional enrichment was detected using the score-based algorithm GSEA ${ }^{70,71}$ considering $\log _{2}$ fold changes as scores and GO terms were grouped to representative GOs using REVIGO ${ }^{72}$. Non-canonical database for GoSeq was first built using Trinotate ${ }^{73}$. Heatmaps of significantly expressed transcripts within selected terms were generated using the heatmap. 2 function from package GPLOTS (v2.17.0). In addition, networks and connections between transcripts were produced using KEGG pathway map (https://www.kegg.jp) ${ }^{74}$ and STRING (https://string-db.org/).

Real-time qPCR. cDNA was synthesized using High Capacity cDNA Reverse Transcription Kit (Applied Biosystems) following manufacturer's protocol without RNase inhibitor and modification of Step two in the PCR reaction program, which lasted $60 \mathrm{~min}$. Primers were designed based on the exon-exon junction using Primer Express 3.0 software (Applied Biosystems) for a set of 10 transcripts of interest, which were significantly up-or down-regulated in the RNA-seq experiment (sequences were obtained from the published Nematostella JGI database https://mycocosm.jgi.doe.gov/Nemve1). Primer sequences used in this study are listed in Supplementary Table S3.

The qPCR reaction was performed using StepOnePluse (Applied Biosystems) 96-well machine with $5 \mu$ Fast SYBR $^{\mathrm{mx}}$ Green Master Mix (Applied Biosystems), $0.5 \mu \mathrm{l}$ of $10 \mu \mathrm{M}$ primers and $25 \mathrm{ng}$ cDNA template in a $10 \mu \mathrm{l}$ volume, using reagent samples without cDNA as negative controls as described before ${ }^{75}$. The thermal profile was set to $95^{\circ} \mathrm{C}$ for $20 \mathrm{~s}$, followed by 40 amplification cycles of $95^{\circ} \mathrm{C}$ for $3 \mathrm{~s}, 60^{\circ} \mathrm{C}$ for $30 \mathrm{~s}$, dissociation cycle of $95^{\circ} \mathrm{C}$ for $15 \mathrm{~s}$ and $60^{\circ} \mathrm{C}$ for $1 \mathrm{~min}$ and then brought back to $95^{\circ} \mathrm{C}$. FUE (far upstream element-binding protein 3 ) transcript (244532) was used as an internal reference control. The constant expression of the FUE level was verified across treatments by qPCR analysis of reactions loaded with equal amounts of cDNA. The quantification analysis was done by the comparative CT method ${ }^{76}$, and all samples were quantified relatively to time 0 (pre-induction). Results are presented as the means with standard error of four biological replicates. While most data were normally distributed according to the Shapiro-Wilk test, the rest were $\log _{10}$ transformed and retested for normal distribution. The significance of the results was determined using two-sample Student's $t$ test or the nonparametric Mann-Whitney $U$-test for data that were not normally distributed after transformation (SPSS software v. 20). The results were considered statistically significant if the null hypothesis could be rejected at the $p<0.05$ level. 


\section{Data availability}

The sequencing data generated during this study have been deposited to the Sequence Read Archive (SRA), under accession PRJNA612671.

Received: 24 March 2021; Accepted: 13 July 2021

Published online: 29 July 2021

\section{References}

1. Bai, S.-N. The concept of the sexual reproduction cycle and its evolutionary significance. Front. Plant Sci. 6, 11 (2015).

2. Heitman, J. Evolution of sexual reproduction: A view from the Fungal Kingdom supports an evolutionary epoch with sex before sexes. Fungal Biol. Rev. 29, 108-117 (2015).

3. Goodenough, U. \& Heitman, J. Origins of eukaryotic sexual reproduction. Cold Spring Harb. Perspect. Biol. 6, a016154 (2014).

4. Speijer, D., Lukeš, J. \& Eliáš, M. Sex is a ubiquitous, ancient, and inherent attribute of eukaryotic life. Proc. Natl. Acad. Sci. USA 112, 8827-8834 (2015).

5. O’Malley, M. A., Leger, M. M., Wideman, J. G. \& Ruiz-Trillo, I. Concepts of the last eukaryotic common ancestor. Nat. Ecol. Evol. 3, 338-344 (2019).

6. Martindale, M. Q., Pang, K. \& Finnerty, J. R. Investigating the origins of triploblasty: "Mesodermal” gene expression in a diploblastic animal, the sea anemone Nematostella vectensis (phylum, Cnidaria; class, Anthozoa). Development 131, 2463-2474 (2004).

7. Ball, E. E., Hayward, D. C., Saint, R. \& Miller, D. J. A simple plan-cnidarians and the origins of developmental mechanisms. Nat. Rev. Genet. 5, 567-577 (2004).

8. Miller, K. J. \& Ayre, D. J. The role of sexual and asexual reproduction in structuring high latitude populations of the reef coral Pocillopora damicornis. Heredity 92, 557-568 (2004).

9. Baird, A. H., Guest, J. R. \& Willis, B. L. Systematic and biogeographical patterns in the reproductive biology of scleractinian corals. Annu. Rev. Ecol. Evol. Syst. 40, 551-571 (2009).

10. Hagman, D. K., Gittings, S. R. \& Vize, P. D. Fertilization in broadcast-spawning corals of the Flower Garden Banks National Marine Sanctuary. Gulf Mex. Sci. 16, 180-187 (1998).

11. Harrison, P. L. et al. Mass spawning in tropical reef corals. Science 223, 1186-1189 (1984).

12. Dunlap, J. C. Molecular bases for circadian clocks. Cell 96, 271-290 (1999).

13. Pittendrigh, C. S. Temporal organization: Reflections of a Darwinian clock-watcher. Annu. Rev. Physiol. 55, 16-54 (1993).

14. Shearman, L. P. et al. Interacting molecular loops in the mammalian circadian clock. Science 288, 1013-1019 (2000).

15. Babcock, R. C. et al. Synchronous spawnings of 105 scleractinian coral species on the Great Barrier Reef. Mar. Biol. 90, 379-394 (1986)

16. Babcock, R. C., Wills, B. L. \& Simpson, C. J. Mass spawning of corals on a high latitude coral reef. Coral Reefs 13, 161-169 (1994).

17. Kaniewska, P., Alon, S., Karako-Lampert, S., Hoegh-Guldberg, O. \& Levy, O. Signaling cascades and the importance of moonlight in coral broadcast mass spawning. eLife 4, e09991 (2015).

18. Levy, O. et al. Light-responsive cryptochromes from a simple multicellular animal, the coral Acropora millepora. Science 318, 467-470 (2007).

19. Rosenberg, Y., Doniger, T., Harii, S., Sinniger, F. \& Levy, O. Canonical and cellular pathways timing gamete release in Acropora digitifera, Okinawa, Japan. Mol. Ecol. 26, 2698-2710 (2017).

20. Reitzel, A. M., Tarrant, A. M. \& Levy, O. Circadian clocks in the cnidaria: Environmental entrainment, molecular regulation, and organismal outputs. Integr. Comp. Biol. 53, 118-130 (2013).

21. Shoguchi, E., Tanaka, M., Shinzato, C., Kawashima, T. \& Satoh, N. A genome-wide survey of photoreceptor and circadian genes in the coral, Acropora digitifera. Gene 515, 426-431 (2013).

22. Vize, P. D. Transcriptome analysis of the circadian regulatory network in the coral Acropora millepora. Biol Bull 216, $131-137$ (2009).

23. Layden, M. J., Rentzsch, F. \& Röttinger, E. The rise of the starlet sea anemone Nematostella vectensis as a model system to investigate development and regeneration. Wiley Interdiscip. Rev. Dev. Biol. 5, 408-428 (2016).

24. Rentzsch, F. \& Technau, U. Genomics and development of Nematostella vectensis and other anthozoans. Curr. Opin. Genet. Dev. 39, 63-70 (2016).

25. Putnam, N. H. et al. Sea anemone genome reveals ancestral eumetazoan gene repertoire and genomic organization. Science 317, 86-94 (2007).

26. Fritzenwanker, J. H. \& Technau, U. Induction of gametogenesis in the basal cnidarian Nematostella vectensis (Anthozoa). Dev. Genes Evol. 212, 99-103 (2002).

27. Stefanik, D. J., Friedman, L. E. \& Finnerty, J. R. Collecting, rearing, spawning and inducing regeneration of the starlet sea anemone, Nematostella vectensis. Nat. Protoc. 8, 916-923 (2013).

28. Hand, C. \& Uhlinger, K. R. The culture, sexual and asexual reproduction, and growth of the sea anemone Nematostella vectensis. Biol. Bull 182, 169-176 (1992).

29. Darling, J. A. et al. Rising starlet: The starlet sea anemone, Nematostella vectensis. BioEssays 27, 211-221 (2005).

30. Genikhovich, G. \& Technau, U. Induction of spawning in the starlet sea anemone Nematostella vectensis, in vitro fertilization of gametes, and dejellying of zygotes. Cold Spring Harb. Protoc. 2009, pdb.prot5281 (2009).

31. Levitan, S. et al. The making of an embryo in a basal metazoan: Proteomic analysis in the sea anemone Nematostella vectensis. Proteomics 15, 4096-4104 (2015).

32. Eckelbarger, K. J., Hand, C. \& Uhlinger, K. R. Ultrastructural features of the trophonema and oogenesis in the starlet sea anemone, Nematostella vectensis (Edwardsiidae). Invertebr. Biol. 127, 381-395 (2008).

33. Moiseeva, E., Rabinowitz, C., Paz, G. \& Rinkevich, B. Histological study on maturation, fertilization and the state of gonadal region following spawning in the model sea anemone, Nematostella vectensis. PLoS ONE 12, e0182677 (2017).

34. Lamb, T. D., Collin, S. P. \& Pugh, E. N. Evolution of the vertebrate eye: Opsins, photoreceptors, retina and eye cup. Nat. Rev. Neurosci. 8, 960-976 (2007).

35. Quiroga Artigas, G. et al. A G protein-coupled receptor mediates neuropeptide-induced oocyte maturation in the jellyfish Clytia. PLoS Biol. 18, e3000614 (2020).

36. Quiroga Artigas, G. et al. A gonad-expressed opsin mediates light-induced spawning in the jellyfish Clytia. eLife 7, e29555 (2018).

37. Lau, C. G. \& Zukin, R. S. NMDA receptor trafficking in synaptic plasticity and neuropsychiatric disorders. Nat. Rev. Neurosci. 8, 413-426 (2007)

38. Paoletti, P., Bellone, C. \& Zhou, Q. NMDA receptor subunit diversity: Impact on receptor properties, synaptic plasticity and disease. Nat. Rev. Neurosci. 14, 383-400 (2013).

39. Ikeda, M. et al. Circadian dynamics of cytosolic and nuclear $\mathrm{Ca}^{2+}$ in single suprachiasmatic nucleus neurons. Neuron 38, 253-263 (2003).

40. Ikeda, M. Calcium dynamics and circadian rhythms in suprachiasmatic nucleus neurons. Neuroscientist 10, 315-324 (2004). 
41. Oren, M. et al. Profiling molecular and behavioral circadian rhythms in the non-symbiotic sea anemone Nematostella vectensis. Sci. Rep. 5, 11418 (2015).

42. Reitzel, A. M., Behrendt, L. \& Tarrant, A. M. Light entrained rhythmic gene expression in the sea anemone Nematostella vectensis: The evolution of the animal circadian clock. PLoS ONE 5, e12805 (2010).

43. Hendricks, W. D., Byrum, C. A. \& Meyer-Bernstein, E. L. Characterization of circadian behavior in the starlet sea anemone, Nematostella vectensis. PLoS ONE 7, e46843 (2012).

44. Fonjallaz, P., Ossipow, V., Wanner, G. \& Schibler, U. The two PAR leucine zipper proteins, TEF and DBP, display similar circadian and tissue-specific expression, but have different target promoter preferences. EMBO J. 15, 351-362 (1996).

45. Gavriouchkina, D. et al. Thyrotroph embryonic factor regulates light-induced transcription of repair genes in zebrafish embryonic cells. PLoS ONE 5, e12542 (2010).

46. Beaver, L. M. et al. Loss of circadian clock function decreases reproductive fitness in males of Drosophila melanogaster. Proc. Natl. Acad. Sci. USA 99, 2134-2139 (2002).

47. Boden, M. J., Varcoe, T. J. \& Kennaway, D. J. Circadian regulation of reproduction: From gamete to offspring. Prog. Biophys. Mol. Biol. 113, 387-397 (2013).

48. Giebultowicz, J. M., Riemann, J. G., Raina, A. K. \& Ridgway, R. L. Circadian system controlling release of sperm in the insect testes. Science 245, 1098-1100 (1989).

49. Naylor, E. Chronobiology of Marine Organisms (Cambridge University Press, 2010). https://doi.org/10.1017/CBO9780511803567.

50. Leach, W. B. \& Reitzel, A. M. Transcriptional remodelling upon light removal in a model cnidarian: Losses and gains in gene expression. Mol. Ecol. 28, 3413-3426 (2019).

51. Oldach, M. J., Workentine, M., Matz, M. V., Fan, T.-Y. \& Vize, P. D. Transcriptome dynamics over a lunar month in a broadcast spawning acroporid coral. Mol. Ecol. 26, 2514-2526 (2017).

52. Nobes, C. D. \& Hall, A. Rho, rac, and cdc42 GTPases regulate the assembly of multimolecular focal complexes associated with actin stress fibers, lamellipodia, and filopodia. Cell 81, 53-62 (1995).

53. Etienne-Manneville, S. Actin and microtubules in cell motility: Which one is in control?. Traffic 5, 470-477 (2004).

54. Parri, M. \& Chiarugi, P. Rac and Rho GTPases in cancer cell motility control. Cell Commun. Signal. 8, 23 (2010).

55. Piekny, A., Werner, M. \& Glotzer, M. Cytokinesis: Welcome to the Rho zone. Trends Cell Biol. 15, 651-658 (2005).

56. Guilluy, C., Garcia-Mata, R. \& Burridge, K. Rho protein crosstalk: Another social network?. Trends Cell Biol. 21, 718-726 (2011).

57. Chauhan, B. K., Lou, M., Zheng, Y. \& Lang, R. A. Balanced Racl and RhoA activities regulate cell shape and drive invagination morphogenesis in epithelia. Proc. Natl. Acad. Sci. USA 108, 18289-18294 (2011).

58. Friedl, P. \& Wolf, K. Tumour-cell invasion and migration: Diversity and escape mechanisms. Nat. Rev. Cancer 3, 362-374 (2003).

59. Damsky, C. H. \& Werb, Z. Signal transduction by integrin receptors for extracellular matrix: Cooperative processing of extracellular information. Curr. Opin. Cell Biol. 4, 772-781 (1992).

60. Hynes, R. O. Integrins: Bidirectional, allosteric signaling machines. Cell 110, 673-687 (2002).

61. Bonnans, C., Chou, J. \& Werb, Z. Remodelling the extracellular matrix in development and disease. Nat. Rev. Mol. Cell Biol. 15, 786-801 (2014).

62. Karsenty, G. \& Park, R. W. Regulation of type I collagen genes expression. Int. Rev. Immunol. 12, 177-185 (1995).

63. Lotan, T. et al. Evolutionary conservation of the mature oocyte proteome. EuPA Open Proteom. 3, 27-36 (2014).

64. Blanchard, G., Druart, X. \& Kestemont, P. Lipid content and fatty acid composition of target tissues in wild Perca fluviatilis females in relation to hepatic status and gonad maturation. J. Fish Biol. 66, 73-85 (2005).

65. Huynh, M. D., Kitts, D. D., Hu, C. \& Trites, A. W. Comparison of fatty acid profiles of spawning and non-spawning Pacific herring, Clupea harengus pallasi. Comp. Biochem. Physiol. B Biochem. Mol. Biol. 146, 504-511 (2007).

66. Bolger, A. M., Lohse, M. \& Usadel, B. Trimmomatic: A flexible trimmer for Illumina sequence data. Bioinformatics 30, 2114-2120 (2014).

67. Dobin, A. et al. STAR: Ultrafast universal RNA-seq aligner. Bioinformatics 29, 15-21 (2013).

68. Love, M. I., Huber, W. \& Anders, S. Moderated estimation of fold change and dispersion for RNA-seq data with DESeq2. Genome Biol. 15, 550 (2014).

69. Young, M. D., Wakefield, M. J., Smyth, G. K. \& Oshlack, A. Gene ontology analysis for RNA-seq: Accounting for selection bias. Genome Biol. 11, R14 (2010).

70. Sergushichev, A. An algorithm for fast preranked gene set enrichment analysis using cumulative statistic calculation. BioRxiv https://doi.org/10.1101/060012 (2016).

71. Subramanian, A. et al. Gene set enrichment analysis: A knowledge-based approach for interpreting genome-wide expression profiles. Proc. Natl. Acad. Sci. USA 102, 15545-15550 (2005).

72. Supek, F., Bošnjak, M., Škunca, N. \& Šmuc, T. REVIGO summarizes and visualizes long lists of gene ontology terms. PLoS ONE 6, e21800 (2011).

73. Bryant, D. M. et al. A tissue-mapped axolotl de novo transcriptome enables identification of limb regeneration factors. Cell Rep. 18, 762-776 (2017).

74. Kanehisa, M., Sato, Y., Kawashima, M., Furumichi, M. \& Tanabe, M. KEGG as a reference resource for gene and protein annotation. Nucleic Acids Res. 44, D457-D462 (2016).

75. Elran, R. et al. Early and late response of Nematostella vectensis transcriptome to heavy metals. Mol. Ecol. 23, 4722-4736 (2014).

76. Schmittgen, T. D. \& Livak, K. J. Analyzing real-time PCR data by the comparative C(T) method. Nat. Protoc. 3, 1101-1108 (2008).

\section{Acknowledgements}

We thank the Bioinformatics Service Unit at the University of Haifa and specifically M. Ofek-Lalzar for her assistance. This study represents partial fulfillment of the requirements for a Ph.D. thesis for M. Rinsky at the Faculty of Life Sciences Bar-Ilan University, Israel.

\section{Author contributions}

S.R. designed and performed the experiments with the assistance of V.B., M.R., S.R., O.L., and T.L. analyzed the data, A.M. performed the bioinformatics, O.L. and T.L. conceived and supervised the project, M.R. wrote the manuscript with input from all authors. All authors discussed the results and commented on the manuscript. All authors read and approved the final version of the manuscript.

\section{Funding}

This work was supported by the Moore Foundation "Unwinding the Circadian Clock in a Sea Anemone" (Grant number 4598 to O.L.). 


\section{Competing interests}

The authors declare no competing interests.

\section{Additional information}

Supplementary Information The online version contains supplementary material available at https://doi.org/ 10.1038/s41598-021-95033-3.

Correspondence and requests for materials should be addressed to O.L. or T.L.

Reprints and permissions information is available at www.nature.com/reprints.

Publisher's note Springer Nature remains neutral with regard to jurisdictional claims in published maps and institutional affiliations.

(c) (1) Open Access This article is licensed under a Creative Commons Attribution 4.0 International License, which permits use, sharing, adaptation, distribution and reproduction in any medium or format, as long as you give appropriate credit to the original author(s) and the source, provide a link to the Creative Commons licence, and indicate if changes were made. The images or other third party material in this article are included in the article's Creative Commons licence, unless indicated otherwise in a credit line to the material. If material is not included in the article's Creative Commons licence and your intended use is not permitted by statutory regulation or exceeds the permitted use, you will need to obtain permission directly from the copyright holder. To view a copy of this licence, visit http://creativecommons.org/licenses/by/4.0/.

(C) The Author(s) 2021 